\title{
Adolescent Social Stress Produces an Enduring Activation of the Rat Locus Coeruleus and Alters its Coherence with the Prefrontal Cortex
}

\author{
Gerard A Zitnik', Andrè L Curtis', Susan K Wood², Jay Arner' and Rita J Valentino*,I \\ 'Division of Stress Neurobiology, Department of Anesthesiology, Abramson Research Center, The Children's Hospital of Philadelphia, Philadelphia, \\ PA, USA; ${ }^{2}$ Department of Pharmacology, Physiology and Neuroscience, University of South Carolina School of Medicine, Columbia, SC, USA
}

\begin{abstract}
Early life stress is associated with the development of psychiatric disorders. Because the locus coeruleus-norepinephrine (LC-NE) system is a major stress-response system that is implicated in psychopathology, developmental differences in the response of this system to stress may contribute to increased vulnerability. Here LC single unit and network activity were compared between adult and adolescent rats during resident-intruder stress. In some rats, LC and medial prefrontal cortex (mPFC) coherence was quantified. The initial stress tonically activated LC neurons and induced theta oscillations, while simultaneously decreasing LC auditory-evoked responses in both age groups. Stress increased LC-mPFC coherence within the theta range. With repeated exposures, adolescent LC neuronal and network activity remained elevated even in the absence of the stressor and were unresponsive to stressor presentation. In contrast, LC neurons of adult rats exposed to repeated social stress were relatively inhibited in the absence of the stressor and mounted robust responses upon stressor presentation. LC sensory-evoked responses were selectively blunted in adolescent rats exposed to repeated social stress. Finally, repeated stress decreased LC-mPFC coherence in the high frequency range (beta and gamma) while maintaining strong coherence in the theta range, selectively in adolescents. Together, these results suggest that adaptive mechanisms that promote stress recovery and maintain basal activity of the brain norepinephrine system in the absence of stress are not fully developed or are vulnerable stress-induced impairments in adolescence. The resulting sustained activation of the LC-NE system after repeated social stress may adversely impact cognition and future social behavior of adolescents.
\end{abstract}

Neuropsychopharmacology (2016) 4I, 1376-1385; doi:I0.1038/npp.2015.289; published online 7 October 2015

\section{INTRODUCTION}

Adolescence is a developmental period of increased stress vulnerability because of the ongoing neuroplasticity (Compas et al, 1993; McCormick and Mathews, 2007) and the immaturity of adaptive processes that are necessary for coping (Gunnar et al, 2009; McCormick and Mathews, 2010; McCormick et al, 2010). For individuals exposed to early life stress, the inability to appropriately adapt to stressors could contribute to the development of psychiatric disorders in adulthood (Halligan et al, 2007; Lupien et al, 2009).

Stressors of a social nature are common and particularly relevant during adolescence as this is a time of dynamic social growth (Gladstone et al, 2006; Sebastian et al, 2010). Human social stress is modeled in rodents using the resident-intruder model (Miczek, 1979). Adult male rats repeatedly exposed to resident-intruder stress display

* Correspondence: Dr RJ Valentino, Division of Stress Neurobiology, Department of Anesthesiology and Critical Care Medicine, Abramson Research Center, Children's Hospital of Philadelphia, 402C, 3416 Civic Center Boulevard, Philadelphia, PA 19104, USA, Tel: + I 2155900650 , Fax: + I 215590 0654, E-mail: valentino@email.chop.edu

Received 25 June 20 I5; revised 24 August 20 I5; accepted 8 September 2015; accepted article preview online II September 2015 endocrine, autonomic and behavioral features that characterize mood disorders in humans, underscoring the potential of this model to produce correlates of stress-related psychiatric diseases (Covington and Miczek, 2005; Covington et al, 2005; Rygula et al, 2005; Wood et al, 2010, 2012, 2013). Although less well studied, adolescent male rats exposed to repeated resident-intruder stress have been reported to have cognitive impairments and escalated cocaine self-administration when tested in adulthood (Burke and Miczek, 2015; Novick et al, 2013; Snyder et al, 2015).

A major stress-response system that is sensitive to social stress is the locus coeruleus-norepinephrine (LC-NE) system (Chaijale et al, 2013). Stressors activate the LC-NE system and this translates to heightened arousal and cognitive changes that are adaptive in the acute stress response (Valentino and Van Bockstaele, 2008). This activation is mediated by corticotropin-releasing factor (CRF), which enhances arousal and cognitive flexibility (Snyder et al, 2012; Valentino and Van Bockstaele, 2008). Acute stress also engages an opioid receptor-mediated inhibition that restrains the excitation and facilitates the recovery of LC activity after stressor termination (Curtis et al, 2001, 2012). Repeated resident-intruder exposure produces enduring changes in 
afferent regulation of the LC that are expressed days after the last stress. Specifically, there is evidence for enhanced opioid drive to the LC in adult male rats (Chaijale et al, 2013). In contrast, LC neurons of early adolescent rats recorded in the anesthetized state $24 \mathrm{~h}$ after the last resident-intruder stress were tonically activated, suggesting age-related differences in stress adaptation (Bingham et al, 2011).

The present study aimed to determine how LC neurons of unanesthetized rats respond to the presence of social stress, whether this changes between the initial and a repeated exposure, and whether these effects are age dependent. Spontaneous activity was recorded from adolescent and adult rats before and during exposure to resident-intruder stress on the first and fifth consecutive day of exposure. Because LC neuronal responses to sensory stimuli are implicated in sensory processing and attention, the effects of residentintruder stress on LC auditory-evoked activity were also compared between age groups (Berridge and Waterhouse, 2003). Additionally, because LC projections to the medial prefrontal cortex (mPFC) have been implicated in the cognitive aspects of stress (Arnsten, 2000), coherence between the LC and MPFC was assessed.

\section{MATERIALS AND METHODS}

\section{Animals}

Male adult (PND 70-83) or adolescent (PND 42-55) Sprague-Dawley rats were used as intruders and male Long-Evans retired male breeders (Charles River, Wilmington, MA) were used as residents. Rats were maintained in a temperature and light controlled environment $\left(20^{\circ} \mathrm{C}, 12-\mathrm{h}\right.$ light-dark cycle) with food and water available ad libitum. Residents were housed individually and intruders were housed two/cage until the day of surgery. Experiments were performed in the light cycle. The care and use of animals were approved by the Children's Hospital of Philadelphia Institutional Animal Care and Use Committee.

\section{Surgery}

Surgery for LC implantation of an 8-microwire electrode array (NB Labs, Denison, TX) was identical to that previously described (Curtis et al, 2012). The array was attached to a Microstar head stage connected to a data acquisition system (AlphaLab; Alpha Omega; Nazareth Illi, Israel). Some rats had an additional depth electrode (tungsten microelectrode, $250 \mu \mathrm{m}$ diameter) implanted into the $\mathrm{mPFC}(+3.2 \mathrm{AP},-0.6 \mathrm{ML},-3.0 \mathrm{DV})$ for simultaneous LC and $\mathrm{mPFC}$ local field potential (LFP) recordings. Animals were allowed 3 days to recover before experimental manipulations.

\section{Repeated Social Stress}

Following post-surgical recovery, rats were exposed to either resident-intruder stress (Miczek, 1979) or control manipulation, as described previously (Wood et al, 2010). Intruders were placed into the resident's cage. When the intruder assumed a supine posture for $3 \mathrm{~s}$ (defeat), a mesh wire barrier was introduced to physically separate the intruder and resident for the remainder of the $30-\mathrm{min}$ session. If the intruder did not assume the defeat posture, then the barrier inserted after $15 \mathrm{~min}$ had elapsed from the time when the intruder was placed into the cage. Control rats were placed in a novel cage for $30 \mathrm{~min}$ daily, with $15 \mathrm{~min}$ behind the wire partition. Rats were returned to home cages following each session. This was repeated for 5 consecutive days.

\section{Electrophysiological Recordings}

Recordings were performed on the first and fifth days of the experimental manipulation. Cables connected the head stage to the data acquisition system and baseline recordings were done in the intruder's home cage. LC multiunit activity was recorded using the AlphaLab (Alpha Omega, Alpharetta, GA), as previously described (Chaijale et al, 2013). LC spontaneous activity was recorded for $300 \mathrm{~s}$ followed by a 200 -s period of auditory stimulation ( $3 \mathrm{kHz}$ tone, $50 \mathrm{~ms}$ duration, $80 \mathrm{db}$ intensity, presented at $0.25 \mathrm{~Hz}$, total 50 presentations). After the trial of auditory stimulation, LC spontaneous discharge rate was recorded for an additional 300 s. The cables were then disconnected and the rat was placed in the resident's cage (or novel cage for controls), as described above. Stress recordings occurred after the physical interaction, while the intruder was in resident's cage but was physically separated from the resident by the wire partition, which maintained visual, olfactory, and auditory communication between resident and intruder.

\section{Electrophysiological Analysis}

Single unit LC waveforms were discriminated and sorted using the WaveMark template-matching algorithm in Spike2 (Supplementary Figures S1A and B) (Cambridge Electronic Design, CED, v7.09), as described previously (Curtis et al, 2012). Determination of single LC neuron waveform templates was made using baseline activity. These templates were matched to the spike train recorded during stressor exposure. This allowed direct comparison of the same single units before and during stress. This template discrimination and matching was repeated on raw spike train files recorded on Day 5. For analysis of LC spontaneous discharge, 'baseline' was the average of the mean rates recorded $300 \mathrm{~s}$ before and after presentation of the auditory stimulus while the intruder was in their homecage. 'Stress' values were calculated using the same time period after placement behind the barrier in the resident's homecage.

Peristimulus time histograms (PSTHs) of activity recorded during repeated auditory stimulation were generated offline. Single LC unit discharge was analyzed using $8 \mathrm{~ms}$ bins beginning $0.5 \mathrm{~s}$ before to $1.5 \mathrm{~s}$ after auditory stimulation. The evoked response was defined as that period after the stimulus when LC discharge rate exceeds 2 SD above the tonic rate until three consecutive bins 2 SD below the tonic rate. Evoked activity was quantified as the sum (total) number of counts (spikes) recorded over the duration of the auditoryevoked response.

LC LFPs were obtained from one of the wires of the multiwire bundle $(12000 \mathrm{kHz}$, bandwidth of $1-500 \mathrm{~Hz})$. Electrode recordings in the $\mathrm{mPFC}$ were amplified at a gain of $5000 \mathrm{~Hz}$, bandwidth of 1-150 Hz. LC-LFP and mPFC raw traces were time stamped in Spike2 to remove noise and converted to Power Spectra Density (PSD) plots indicating 
the relative power in 128 frequency bins from 0 to $50 \mathrm{~Hz}$ using Neuroexplorer (Nex Technologies, Madison, AL).

\section{Histology}

After the last experimental manipulation, rats were deeply anesthetized with isofluorane and current $(17 \mu \mathrm{A}$ for $20 \mathrm{~s})$ was passed through the channel(s) on which single unit activity was observed. Rats were perfused with $6 \%$ potassium ferrocyanide, forming a Prussian blue reaction for identification of the electrode site (Supplementary Figure S1C). Histological verification of electrode placement in the mPFC was also confirmed postmortem.

\section{Statistical Analysis}

Two-way repeated measures analysis of variance (rmANOVA) with age (adolescent $v s$ adult) and condition (stress $v s$ control) as between-subject factor and time (pre $v s$ poststress) as the within-subject factor were used for comparisons between treatment groups on either day 1 or day 5 . Two-way rmANOVA was used to compare changes in spontaneous and evoked LC discharge between control and stressed rats pre- and post-stress within each age group (condition as between-subject factor and time as the withinsubject factor). Two-way ANOVAs (condition as betweensubject factor and day as the within-subject factor) were used to compare mean LC discharge rates at baseline and during stress within each treatment group on day $5 v s$ day 1 , with Student-Newman-Keuls for post hoc comparisons between means.

For LFP analysis, the average PSD value for different frequency bands (delta, 2-4 Hz; theta, 6-8 Hz; alpha, $8-12 \mathrm{~Hz}$; and beta, $12-20 \mathrm{~Hz}$ ) was calculated. These values were compared between days by a mixed-factor ANOVA (age and condition as the between-subject factor and frequency band as the repeated measure), with follow-up analysis determined using Tukey's HSD for post hoc comparisons between means. LC-mPFC coherence was calculated in Neuroexplorer using the LFP from a channel of the LC multiwire bundle and the LFP recorded from the depth electrode in the mPFC. LFP and coherence data were converted to spectrograms and heat maps, respectively, using a custom-written MATLAB program (MathWorks, Natick, MA).

\section{RESULTS}

\section{LC Spontaneous Activity}

On day 1 before any manipulation, mean LC spontaneous discharge rates were comparable between adult $(1.7 \pm 0.2 \mathrm{~Hz}$, $n=32$ cells $/ 8$ rats $)$ and adolescent $(1.4 \pm 0.3 \mathrm{~Hz}, n=26$ cells/ 7 rats) rats $(\mathrm{F}(1,56)=0.556 ; p=0.46)$. Initial exposure to resident-intruder stress increased LC discharge rates of both adults and adolescents (Figure 1a; Table 1). A two-way rmANOVA revealed that the change in LC discharge among stressed animals was significantly different from controls on day 1 (Time $\times$ Condition; $F(1,54)=17.92 ; p<0.001$ ), indicating an effect of stress in both age groups that was not different between age groups (Time $\times$ Condition $\times$ Age; $\mathrm{F}(1,54)=0.016 ; p=0.901)$.
After five exposures to resident-intruder stress, adolescent LC discharge rates were elevated in the absence of stress to levels that were comparable to those seen during the initial exposure to resident-intruder stress (Table 1, $t=0.67$; upper CL diff $=0.95$, lower CL diff $=-0.48$ ). The mean baseline spontaneous LC discharge rate was significantly higher on day 5 vs day 1 in adolescent animals (Condition $\times$ Day; $F$ $(3,43)=3.804 ; p=0.017)$ (Figure 1b1; Table 1). Exposure of these rats to resident-intruder stress did not further elevate LC activity (Figure 1c1; Table 1). In contrast, LC discharge rates of adult rats before stress on the fifth day were relatively inhibited compared with their pre-stress rates on the first day of stress (Condition $\times$ Day; $F(3,51)=3.022 ; p=0.038$ ) (Figure 1b2; Table 1). Notably, LC neurons of adult rats exposed to repeated social stress remained strongly responsive to the stress (Figure 1c2; Table 1). Within-subject comparison revealed that stress altered spontaneous LC activity differently between age groups on day 5 (Time $\times$ Condition $\times$ Age; $\mathrm{F}(1,39)=9.221 ; p=0.004)$.

\section{LC Auditory-Evoked Activity}

As previously described, LC neurons of unanesthetized rats were robustly activated by discrete auditory stimuli (AstonJones and Bloom, 1981). Figure 2 shows PSTHs of single unit LC activity during presentation of the auditory stimulus before and during social stress for an adult and adolescent rat on day 1 and day 5. LC auditory-evoked discharge was decreased during the initial stress exposure (Figure 2; Table 1). An rmANOVA revealed that the change in LC discharge among stressed animals was significantly different from controls on day 1 (Time $\times$ Condition $\mathrm{F}(1,48)=6.523$; $p=0.014)$, indicating an effect of stress in both age groups that was not different between age groups (Time $\times$ Condition $\times$ Age; $\mathrm{F}(1,48)=1.109 ; p=0.298)$. In contrast, by the fifth social stress exposure, the evoked response recovered in adults but remained inhibited in adolescent rats (Figure 2; Table 1). An rmANOVA revealed that stress altered LC auditory-evoked activity differently between age groups on day $5($ Time $\times$ Condition $\times$ Age; $F(1,37)=9.016 ; p=0.005)$.

\section{LC Network Activity and LC-Prefrontal Cortical Coherence}

LC network activity was recorded as LFPs in a population of adolescent ( $n=8$ for days 1 and 5) and adult (day 1: $n=5$, day 5: $n=4$ ) rats during social stress exposure. Spectrograms displaying power in different frequencies over time show that LC LFPs are characterized by distinct high power in delta and/or theta frequencies depending on age and experimental condition (Figure 3). On the first day of the experiment before stress, both adolescents and adults display prominent activity in the delta and theta frequency range (Figure $3 a$ ). There was an effect of frequency $(\mathrm{F}(3,12)=829 ; p<0.001)$ that was not different between age groups (Frequency $\times$ Age $(\mathrm{F}(3,12)=2.358 ; p=0.123)$. Resident-intruder exposure elicited a robust theta oscillation in both adults and adolescents, and decreased power in lower (delta) and higher (beta) frequencies (Figure 3a). A two-way rmANOVA revealed a main effect $(\mathrm{F}(3,22)=3.69 ; p=0.027)$ and effect of stress $($ Frequency $\times$ Time; $(\mathrm{F}(3,20)=4.341 ; p=0.016)$ that was not different between age groups (Frequency $\times$ Time $\times$ Age; 
a1

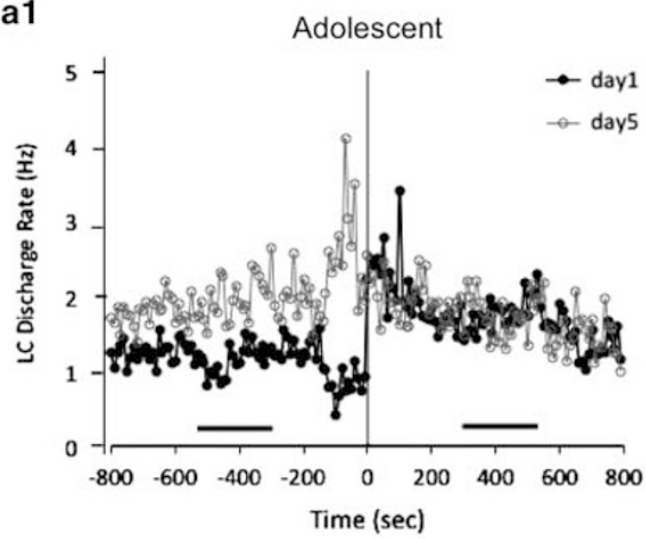

b1

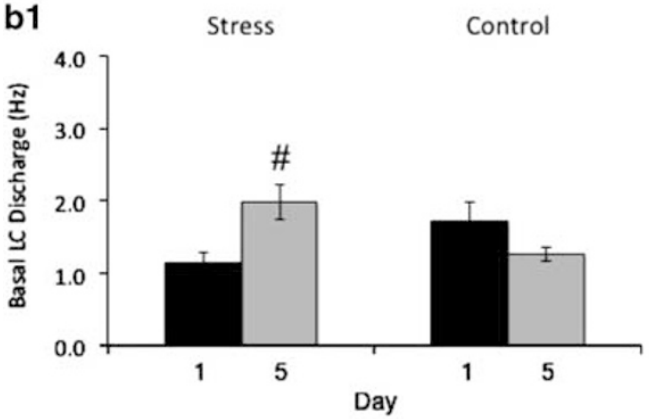

c1

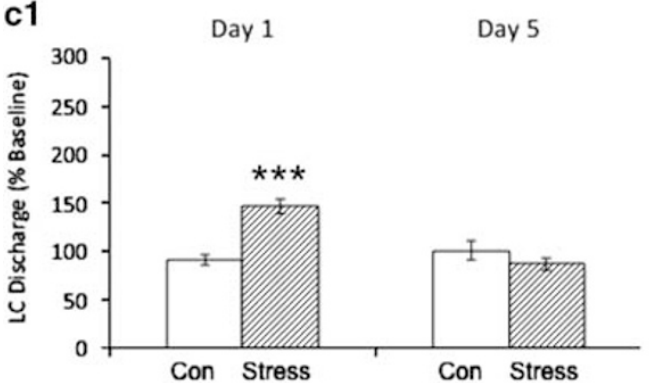

a2

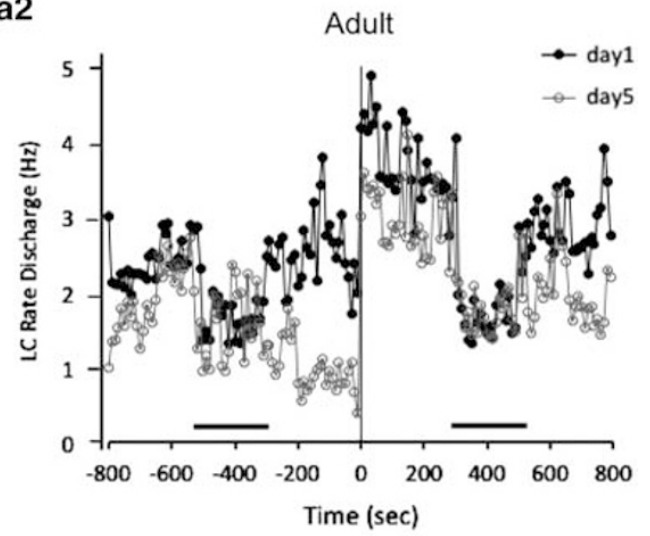

b2 Stress Control

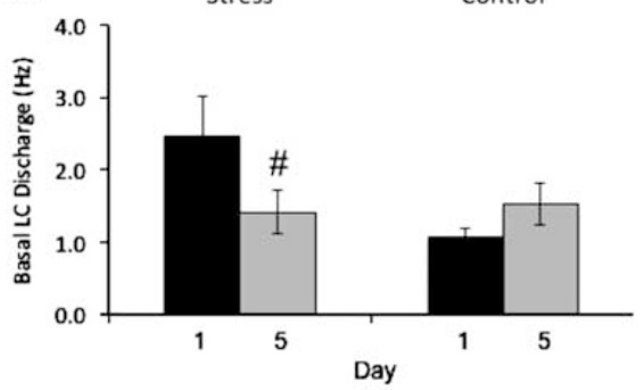

c2

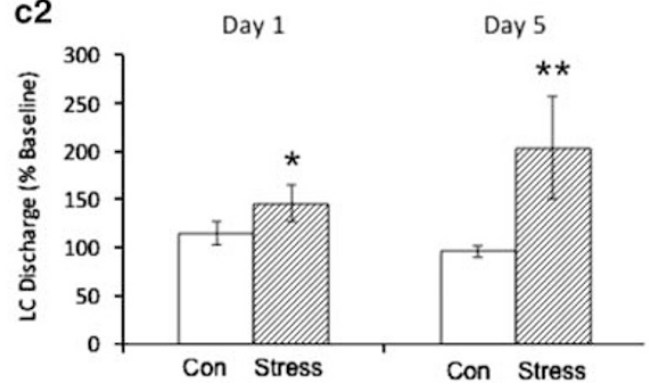

Figure I Age-dependent effects of social stress on LC spontaneous discharge. (a) Line graphs showing the mean LC discharge rates over time on day I (black, filled) and day 5 (gray, unfilled) for adolescent (al) and adult (a2) rats undergoing social stress. The abscissae represent time (seconds) and the ordinates indicate the mean LC discharge rate $(\mathrm{Hz})$. Time zero represents the start of recording during stressor exposure as described in Materials and Methods. Horizontal black bars represent the time of auditory stimulation. Note the increased spontaneous LC discharge rate following exposure to the stressor on day I in both the adolescent and adult animals. Also note that basal rates in adolescent animals are heightened on day 5 vs day I, with exposure to the stressor producing no change in spontaneous activity from baseline. Conversely, adults display lower spontaneous discharge LC rates at baseline on day 5 vs day I, with exposure to the stressor producing a robust increase in activity on day 5. (b) Bar graphs showing the mean baseline spontaneous LC discharge rate in adolescent (bl) and adult (b2) rats on day I (black) and day 5 (gray). ${ }^{\#} p<0.05$; post hoc comparisons to day I values. Error bars represent \pm I SEM. (c) Bar graphs showing the change in LC discharge rates from baseline (expressed as a percentage of the baseline rate) in adolescent ( $\mathrm{cl}$ ) and adult (c2) control (unfilled) and stress rats (hatched) on days I and 5. Rates are based on the mean firing rate $(\mathrm{Hz})$ from the two 5-min periods of spontaneous recording at baseline or during stress. ${ }^{*} p<0.05$, ${ }^{*} p p<0.01$, ${ }^{*} * * * 0.00$ I; two-way rmANOVA comparison $\mathrm{w} /$ controls. Error bars represent \pm I SEM.

$\mathrm{F}(3,20)=0.545 ; p=0.657)$. Two-way rmANOVAs analyzing the change within specific frequency bands confirmed that stress decreased power in delta $(\mathrm{F}(1,11)=9.074 ; p=0.012)$ and beta $(\mathrm{F}(1,11)=15.26 ; p=0.002)$ frequencies and increased theta $(\mathrm{F}(1,11)=14.69 ; p=0.003)$ oscillations in both age groups. On day 5 , exposure to the stressor had no effect on LC LFP frequency (Frequency $\times$ Time; $\mathrm{F}(3,18)=0.482$; $p=0.699$ ) (Figure $3 b)$. However, there was an overall effect of age $(\mathrm{F}(1,20)=10.73 ; p=0.004)$ and a Frequency $\times$ Age interaction $(\mathrm{F}(3,18)=4.995 ; p=0.011)$. A bias in power toward delta frequency distinguished adults $(\mathrm{F}(1,20)=5.92$; $p=0.025)$, whereas adolescents were distinguished by theta oscillations $(\mathrm{F}(1,20)=15.94 ; p=0.001)$ (Figure 3b2). Notably, a comparison between the first and fifth stressor experience indicated no difference in LC network activity for adults $(\mathrm{F}(3,12)=1.294 ; p=0.321)$. In contrast, for adolescents there was a Frequency $\times$ Day $\times$ Time interaction $(F(3,26)=3.442$; $p=0.031$ ), indicating that stress enhanced theta frequency on day 1 only because on day 5 LC network activity already showed prominent theta oscillations before the stress $(\mathrm{F}(3,28)=5.623 ; p=0.004)$.

In rats in which both LC and mPFC LFP recordings were optimal ( $n=4$ /group), a strong coherence was detected in two specific frequency bands, $2-3 \mathrm{~Hz}$ and $7-9 \mathrm{~Hz}$ 
Table I Absolute Mean LC Discharge Rates for Each Experimental Condition

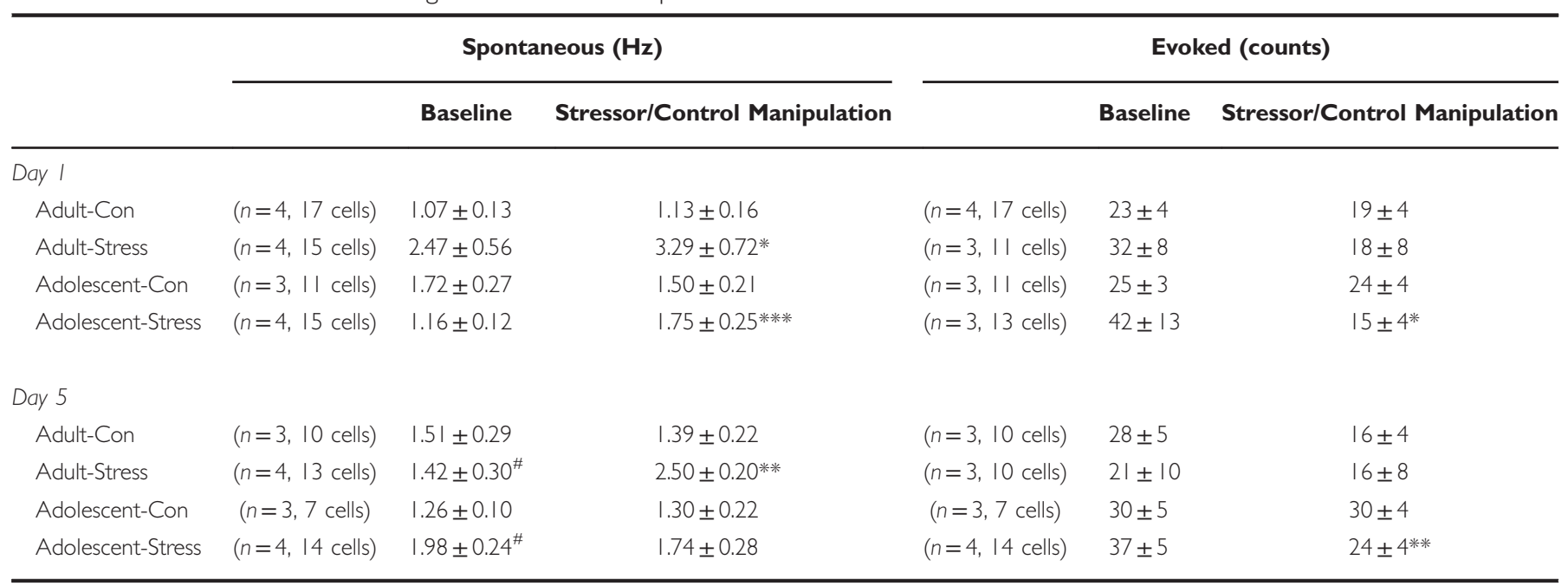

Absolute mean LC spontaneous discharge and sensory evoked activity at baseline and during exposure to the social stressor (or control manipulation) in adolescent and adult animals on days I and 5. Animal and cell numbers for each value are provided. $* p<0.05$, $* *$ $p<0.0$ I, $* * * * 0.00$ I; two-way rmANOVA comparison w/ controls. ${ }^{\#} p<0.05$; post hoc comparison w/ day I values.

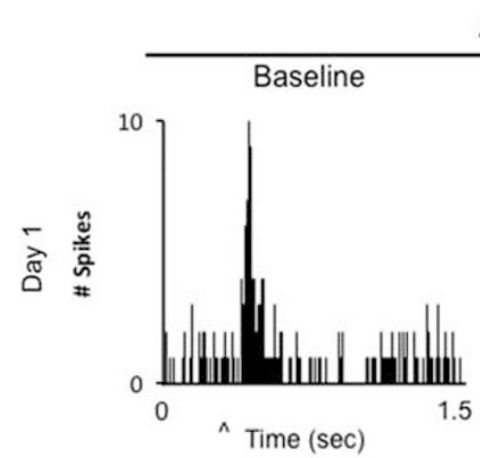

Adult

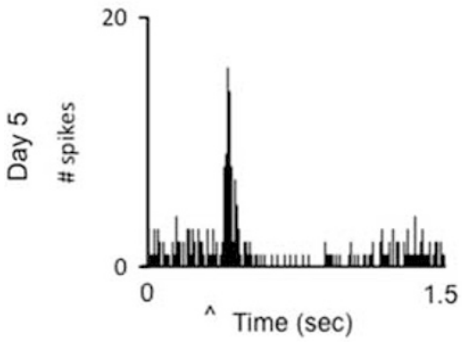

Stress

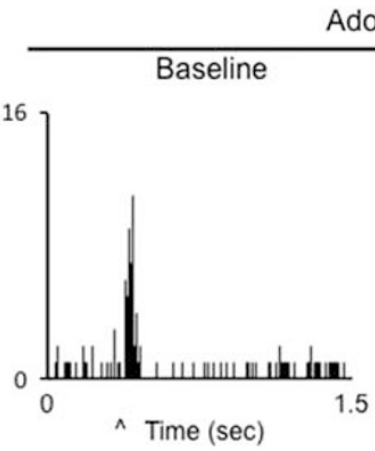

Adolescent
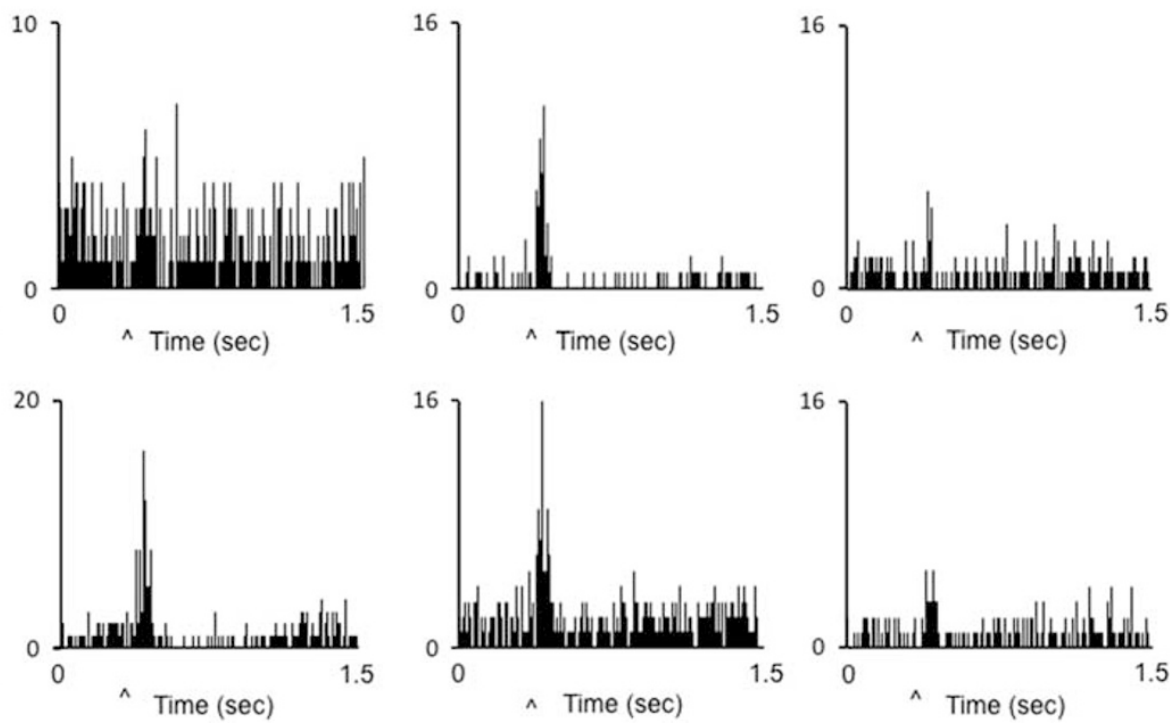

Figure 2 Age-dependent effects of social stress on LC auditory-evoked activity. Example peristimulus time histograms (PSTHs) of single LC neurons generated during repeated presentations of the auditory stimulus before (baseline) and during social stress on days I and 5 in adult and adolescent animals. Spikes were generated in $8 \mathrm{~ms}$ bins. The ordinates represent LC discharge (counts). The abscissae represent time, ranging from $0.5 \mathrm{~s}$ before presentation of the auditory stimulus to $1.5 \mathrm{~s}$ afterwards. ' ${ }^{\prime}$ ' denotes presentation of the auditory stimulus. Note the diminished sensory-evoked response from baseline in both age groups on day I, and in adolescents only on day 5.

(Figure 4b). Social stress exposure decreased LC-mPFC coherence within the $2-3 \mathrm{~Hz}$ band (Time; $\mathrm{F}(1,11)=5.294$; $p=0.042)$ regardless of day (Time $\times$ Day; $\mathrm{F}(1,11)=0.001$; $p=0.976)$ or age (Time $\times$ Age $\mathrm{F}(1,11)=0.079 ; p=0.785)$ (Figure 4c). At the same time, stress exposure increased LC-mPFC coherence within the $7-9 \mathrm{~Hz}$ range (Time; $\mathrm{F}(1,11)=10.81 ; p=0.007)$ regardless of day (Time $\times$ Day; $\mathrm{F}(1,11)=0.167 ; p=0.69)$ or age (Time $\times$ Age $\mathrm{F}(1,11)=0.605$; $p=0.453$ ) (Figure $4 c$ ). In adolescents only, repeated social stress exposure additionally decreased LC-mPFC coherence at higher frequencies $(12-40 \mathrm{~Hz})$ (Figure $4 \mathrm{~b}$ and $\mathrm{c}$ ). A comparison of coherence on day $1 v s$ day 5 revealed a trend for change among adolescents $(\mathrm{F}(1,12)=4.127$; $p=0.065)$ but not adults $(\mathrm{F}(1,11)=0.7 ; p=0.4)$. The effect in adolescents was attributed to diminished activity within the beta $(12-20 \mathrm{~Hz})$ and gamma $(20-40 \mathrm{~Hz})$ range (Day effect for beta: $\mathrm{F}(1,15)=9.3 ; p=0.01$; Day effect for gamma: $\mathrm{F}(1,15)=7.8 ; p=0.02)$. 
a1
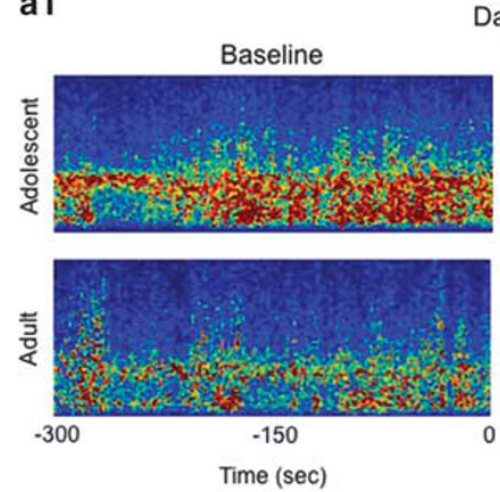

Day 1
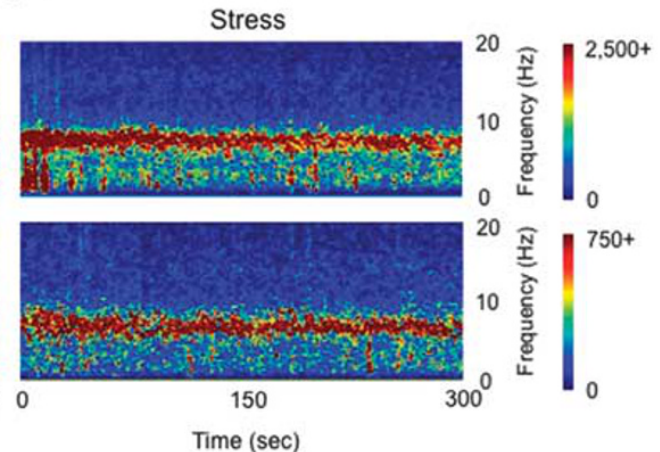

b1
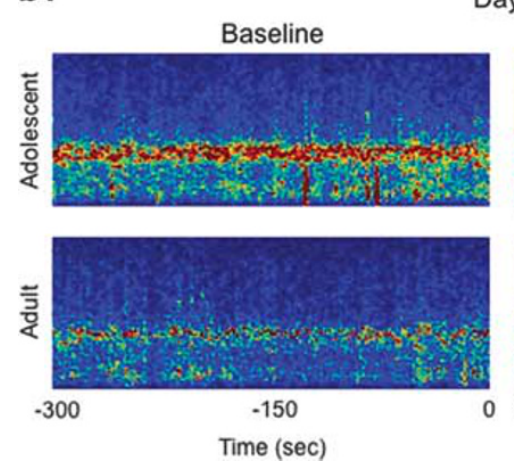

Day 5

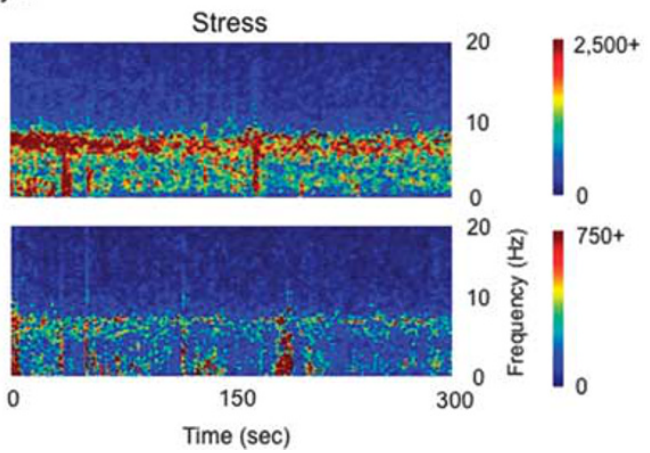

a2

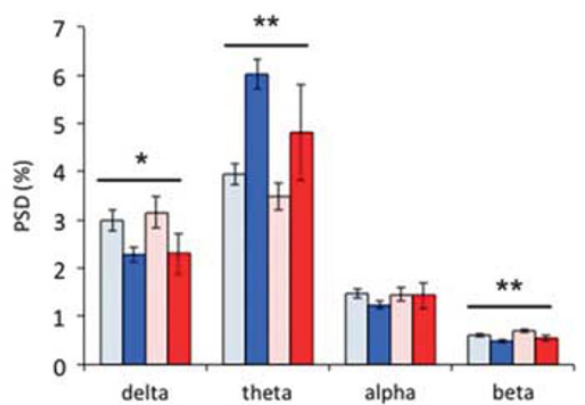

b2

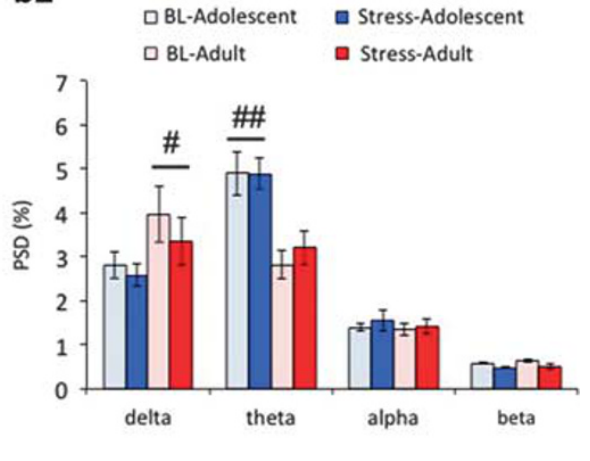

Figure 3 Age-dependent effects of social stress on LC local field potentials (LFPs). (al, b I) Spectrograms displaying the average frequency (ordinates) over time (abscissae) across all animals recorded in each group on day I (al) and day 5 (b I). Baseline is shown as the 300 s immediately before exposure, recorded in the intruder's home cage. Stress is shown as first $300 \mathrm{~s}$ after placement of the partition in the resident's cage. (a2, b2) Corresponding bar graphs represent the mean power spectral density (PSD) values on day I (a2) and day 5 (b2). Error bars represent \pm I SEM. Note the decrease in the power in delta and beta frequencies and the increase in power in the theta frequency range in both age groups after exposure to the stressor on day I. $* * *<0.01$ stress effect; ${ }^{*} p<0.05$ stress effect. Note the overall higher delta activity in adults vs adolescents and overall higher theta activity in adolescents vs adults on day $5 .{ }^{*} p<0.05$ age effect; ${ }^{\# \#} p<0.01$ age effect.

\section{DISCUSSION}

The present study demonstrated distinct effects of social stress on LC activity depending on whether it occurs in adolescence or adulthood. Like other stressors, acute social stress shifted the mode of LC activity toward a high tonic state characterized by increased spontaneous discharge rate and diminished responses to discrete sensory stimuli (Curtis et al, 2001, 2012). Additionally, acute social stress altered LC network activity, inducing prominent theta oscillations within the LC and increasing LC-mPFC coherence within the theta range. Whereas the effects of a single social stress were similar for both adults and adolescents, repeated stress exposure revealed marked age-related differences. Notably, repeated exposure to social stress rendered LC neurons of adolescent rats hyperactive with a sustained theta oscillation in the absence of stress and unresponsive to stress re-exposure. In marked contrast, LC neurons of adult rats were relatively inhibited in the absence of the stressor and remained strongly responsive to a subsequent challenge, suggesting a degree of recovery that is not present in adolescence. In parallel, LC neurons of adults, but not adolescents, adapted to stress-induced decreases of LC auditory-evoked responses. Finally, repeated social stress exposure decreased coherence in the high frequency range in adolescent animals. Together, the findings reveal a muted ability of the LC-NE system of adolescents to recover from repeated social stress. As adolescence is a socially dynamic developmental period, the results underscore the potential for negative social experiences to have impairing effects on behavior and/or cognition because of the unique vulnerability of the brain NE system during this time.

\section{Acute Social Stress and LC Single Unit Activity}

The present study is novel in its characterization of rat LC neuronal activity during social stress and in the comparison with these end points between adolescent and adult rats. The initial exposure to social stress produced the predicted effects to increase spontaneous LC discharge and decrease auditoryevoked discharge regardless of age. This stress-induced shift in LC firing mode to a high tonic state that is less responsive to discrete stimuli is proposed to facilitate a shift to scanning attention and behavioral flexibility, responses that would be adaptive in coping with immediate environmental challenges (Aston-Jones and Cohen, 2005; Valentino and Van Bockstaele, 2008).

\section{Repeated Social Stress and Basal LC Activity}

Striking developmental differences in LC activity were apparent after repeated stress. Repeated social stress and 
a

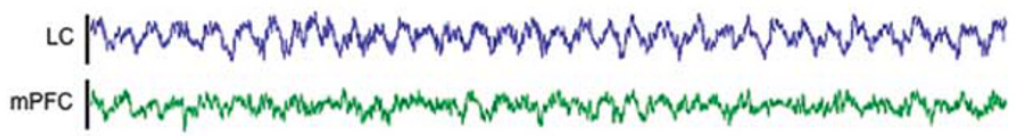

b
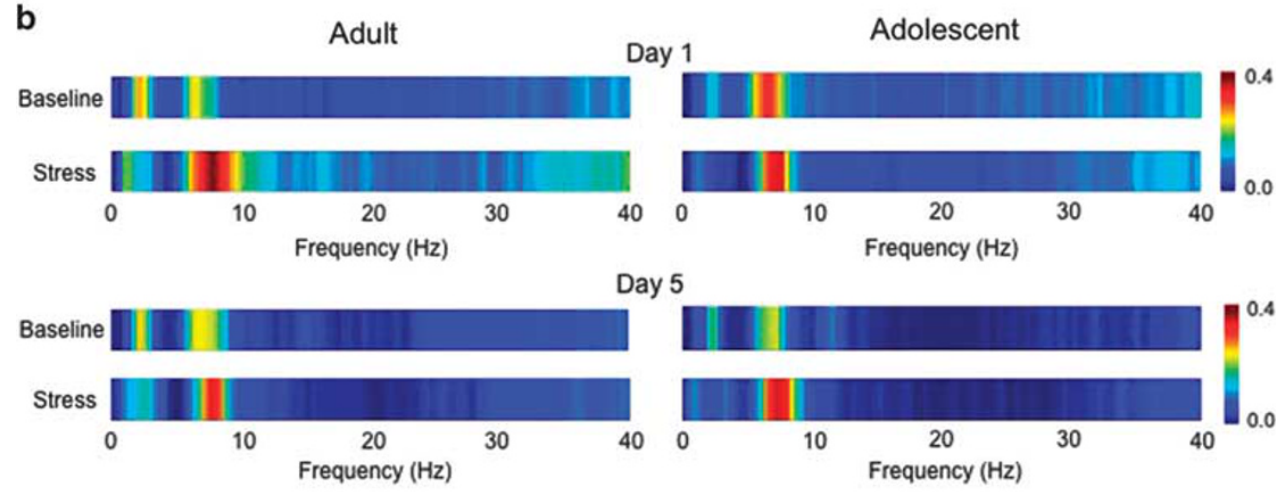

Day 5
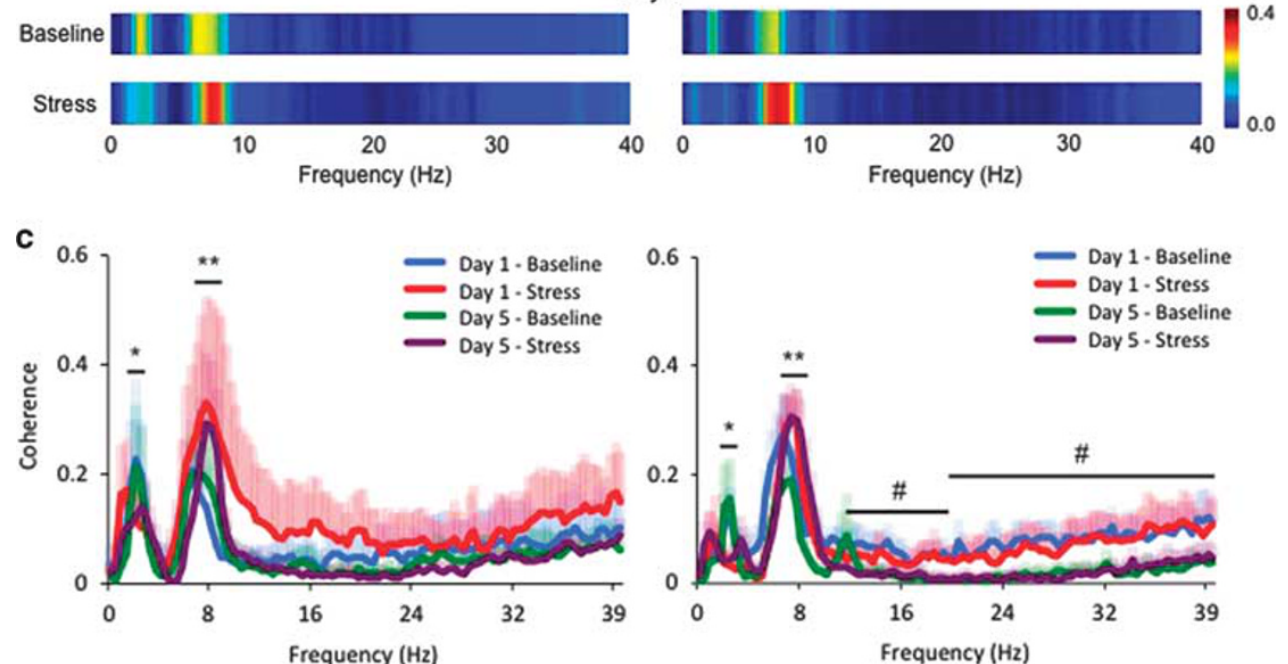

Figure 4 Age-dependent effects of social stress on coherence between the LC and PFC LFPs. (a) Representative raw traces of LFPs in the LC and mPFC simultaneously recorded from an adolescent animal at baseline over a 5-s period. (b) Heat maps displaying the average coherence value at different frequencies recorded in each group on days I and 5. (c) Line plots showing the effect of a single or repeated stress on LC-mPFC coherence in adolescent and adult rats. The ordinates show the mean coherence values and the abscissae indicate different frequencies $(\mathrm{Hz})$. Note the stressor-mediated shift in coherence, increasing coherence between 7 and $9 \mathrm{~Hz}$ and decreasing within the $2-3 \mathrm{~Hz}$ range. Shaded error bars are color matched to the corresponding time/day, representing + I SEM. $* p<0.05$, ** $p<0.0$ I; stress effects. Also note the diminished activity in the high frequency beta $(12-20 \mathrm{~Hz})$ and gamma $(20-40 \mathrm{~Hz})$ range from day $I$ to day 5 in adolescent animals. ${ }^{\#}<0.05$; day effects.

repeated exposure to the single prolonged stress paradigm produce LC hypoactivity in adult rats that is observed days after stressor termination (Chaijale et al, 2013; George et al, 2013). Inhibited LC discharge rate is also seen immediately after acute stress termination or after repeated trials of noise stress (Abercrombie and Jacobs, 1987; Curtis et al, 2001). This post-stress inhibition serves to promote recovery of LC activity after stressor termination and protect against sustained hyperactivity (Curtis et al, 2001). Opioid afferents to the LC and occupation of $\mu$-opioid receptors have been implicated in this inhibition (Curtis et al, 2001, 2012). The current findings indicate that the suppression of LC activity in adult rats exposed to repeated social stress is ongoing by the fifth day of social stress. Importantly, the results suggest that this recovery mechanism is either not fully developed in adolescence or that exposure to stress during this time period impairs this recovery mechanism. Rather, repeated social stress created a condition of LC hyperactivity in the absence of stress in adolescents. These findings are similar to those reported in anesthetized rats in which LC activity was recorded $24 \mathrm{~h}$ after the final exposure to the stressor, although in that study LC hyperactivity was observed in juvenile, but not in mid-adolescent rats (Bingham et al,
2011). However, the present study differed from the latter in that recordings were done in the unanesthetized state and during, rather than after, the resident-intruder stress. The present results imply that in adolescents exposed to repeated social stress the brain noradrenergic system will maintain a high tonic state that is associated with hyperarousal and labile attention. Consistent with this, defensive burying behavior is enhanced in adolescent rats exposed to repeated resident-intruder stress (Bingham et al, 2011). The duration the LC hyperactivity remains to be determined. LC recordings from adult rats that were exposed to repeated residentintruder stress during adolescence suggest that this effect does not endure into adulthood although those recordings were performed in the anesthetized state (Bingham et al, 2011).

\section{Repeated Social Stress and Sensory-Evoked LC Activation}

The ability of a resident-intruder exposure to activate LC neurons in rats with a history of social stress was age dependent, being maintained in adults and lost in adolescents. The lack of activation in adolescents is not likely due to 
a ceiling effect because LC neurons of unanesthetized rats can exhibit frequencies greater than $2 \mathrm{~Hz}$ (see Figure 1). Rather, the results suggest that LC neurons of adolescent rats have not recovered from previous exposures and that the mechanisms by which a single resident-intruder exposure activates LC neurons are already engaged so that subsequent exposures are ineffective.

LC auditory-evoked responses were also altered in an agedependent manner in rats exposed to repeated social stress. Typically, sensory-evoked LC discharge is inhibited when LC spontaneous discharge is tonically elevated, as was observed after the initial stress. The lack of selective responding to a single stimulus would be adaptive in a dynamic environment with life-threatening challenges. Surprisingly, after the fifth stress exposure, auditory-evoked LC discharge of adult rats was unaffected although the stressor elevated LC spontaneous discharge. Release of endogenous opioids that increase the signal-to-noise ratio of LC sensory responses could explain this (Valentino and Wehby, 1988). An unexpected finding in adolescents exposed to repeated social stress was that auditory responses were maintained in the absence of stress although LC discharge was elevated and exposure to stress decreased the auditory response although it did not affect spontaneous activity. Future studies focused on social stress effects on LC afferents in adults and adolescents should elucidate the mechanism underlying these stress-induced changes in LC sensory responses and their age dependency.

\section{Social Stress and LC Network Activity}

LFPs represent the summation of local neuron potentials and provide a read-out of oscillations emerging from the synchronization of neurons (Einevoll et al, 2013; FernandezRuiz and Herreras, 2013). This network activity is thought to be fundamental to cognitive processes. Oscillations of specific frequencies were prominent in the LC depending on age and condition. Notably, the initial exposure to social stress induced a robust theta oscillation in both adult and adolescent LC. Theta oscillations in LC forebrain targets, such as the hippocampus and the amygdala, have been linked to fear consolidation and anxiety and these could arise from LC afferents because regionally selective LC activation is sufficient to drive hippocampal theta oscillations (Adhikari et al, 2010; Berridge and Foote, 1991). As would be predicted by changes in LC single unit activity, theta oscillations were sustained in the LC of adolescent rats that had been repeatedly stressed, even when recorded in the home cage in the absence of stress. These effects on LC network activity reinforce the pronounced effect of repeated social stress in adolescents to sustain an anxiogenic, hyperaroused state.

Evidence for anatomical and functional connectivity between the LC and mPFC (Jodo et al, 1998; Lestienne et al, 1997; Morrison et al, 1979; Sara and Herve-Minvielle, 1995) supported a rationale for examining LC-mPFC coherence, a measure of the synchronous oscillations between brain regions (Engel et al, 2001; Fries, 2005). Our recordings are, to our knowledge, the first to display the striking coherence between the LC and the mPFC in specific frequency bands in the awake, freely moving animal. Notably, stress exposure shifted LC-mPFC coherence to a distinct frequency range $(7-9 \mathrm{~Hz})$ regardless of day or age. As coherence between the $\mathrm{mPFC}$ and other brain regions within the theta range has been implicated in fear memory (Jacinto et al, 2013; Lesting et al, 2013), the current study provides evidence that the LC also participates in this anxiogenic neural circuit. At the same time that repeated stress enhanced LC-mPFC theta coherence, it impaired coherence at higher frequencies (eg, gamma) that have been implicated in higher cognitive processing, selectively in adolescent rats (Benchenane et al, 2011). These changes in LC-mPFC coherence could combine to promote an anxiogenic state with diminished cognitive ability to cope.

\section{Clinical Implications}

The dynamics of social interactions during adolescence shape individual mental health and future social status. Although social stress can occur at all ages, the present study suggested that its impact on the brain NE system would be significantly greater in adolescents compared with adults because of the decreased capacity of the adolescent LC for recovery and adaptation. Given the functions of the LC-NE system, the consequences of tonic hyperactivation would include sleep disturbances, inability to concentrate, behavioral hyperactivity, and anxiety. Previous studies from our laboratory suggest that this elevated basal discharge is associated with increased defensive behaviors (Bingham et al, 2011). Diminished LC sensory responses could additionally impair sensory processing and performance in attention-related tasks. Finally, the inability of the adolescent LC to mount an appropriate response to a subsequent social challenge, taken with decreased LC-cortical gamma coherence could impair the ability to cognitively cope with future social challenges.

\section{FUNDING AND DISCLOSURE}

The authors declare no conflict of interest.

\section{ACKNOWLEDGMENTS}

We acknowledge the technical assistance of Andrew Ho, Calliandra Lombard, and Claire Read. This work was supported by NIMH MH0939810 (RJV), DARPA W911NF1010093, T32 MH014654-36 Training Program in Neuropsychopharmacology (GAZ), a Brain Behavior Research Foundation Young Investigator Award 17830 (SKW) and AHA 13BGIA14370026 (SKW).

\section{REFERENCES}

Abercrombie ED, Jacobs BL (1987). Single-unit response of noradrenergic neurons in the locus coeruleus of freely moving cats. II. Adaptation to chronically presented stressful stimuli. J Neurosci 7: 2844-2848.

Adhikari A, Sigurdsson T, Topiwala MA, Gordon JA (2010). Cross-correlation of instantaneous amplitudes of field potential oscillations: a straightforward method to estimate the directionality and lag between brain areas. J Neurosci Methods 191: 191-200.

Arnsten AF (2000). Stress impairs prefrontal cortical function in rats and monkeys: role of dopamine D1 and norepinephrine alpha-1 receptor mechanisms. Prog Brain Res 126: 183-192. 
Aston-Jones G, Bloom FE (1981). Norepinephrine-containing locus coeruleus neurons in behaving rats exhibit pronounced responses to non-noxious environmental stimuli. J Neurosci 1: 887-900.

Aston-Jones G, Cohen JD (2005). An integrative theory of locus coeruleus-norepinephrine function: adaptive gain and optimal performance. Ann Rev Neurosci 28: 403-450.

Benchenane K, Tiesinga PH, Battaglia FP (2011). Oscillations in the prefrontal cortex: a gateway to memory and attention. Curr Opin Neurobiol 21: 475-485.

Berridge CW, Foote SL (1991). Effects of locus coeruleus activation on electroencephalographic activity in the neocortex and hippocampus. J Neurosci 11: 3135-3145.

Berridge CW, Waterhouse BD (2003). The locus coeruleusnoradrenergic system: modulation of behavioral state and statedependent cognitive processes. Brain Res Brain Res Rev 42: 33-84.

Bingham B, McFadden K, Zhang X, Bhatnagar S, Beck S, Valentino R (2011). Early adolescence as a critical window during which social stress distinctly alters behavior and brain norepinephrine activity. Neuropsychopharmacology 36: 896-909.

Burke AR, Miczek KA (2015). Escalation of cocaine selfadministration in adulthood after social defeat of adolescent rats: role of social experience and adaptive coping behavior. Psychopharmacology (Berl) 232: 3067-3079.

Chaijale NN, Curtis AL, Wood SK, Zhang XY, Bhatnagar S, Reyes BA et al (2013). Social stress engages opioid regulation of locus coeruleus norepinephrine neurons and induces a state of cellular and physical opiate dependence. Neuropsychopharmacology 38: 1833-1843.

Compas BE, Orosan PG, Grant KE (1993). Adolescent stress and coping: implications for psychopathology during adolescence. J Adolesc 16: 331-349.

Covington HE 3rd, Miczek KA (2005). Intense cocaine selfadministration after episodic social defeat stress, but not after aggressive behavior: dissociation from corticosterone activation. Psychopharmacology (Berl) 183: 331-340.

Covington HE 3rd, Kikusui T, Goodhue J, Nikulina EM, Hammer RP Jr., Miczek KA (2005). Brief social defeat stress: long lasting effects on cocaine taking during a binge and zif268 mRNA expression in the amygdala and prefrontal cortex. Neuropsychopharmacology 30: 310-321.

Curtis AL, Bello NT, Valentino RJ (2001). Endogenous opioids in the locus coeruleus function to limit the noradrenergic response to stress. J Neurosci 21: RC152.

Curtis AL, Leiser SC, Snyder K, Valentino RJ (2012). Predator stress engages corticotropin-releasing factor and opioid systems to alter the operating mode of locus coeruleus norepinephrine neurons. Neuropharmacology 62: 1737-1745.

Einevoll GT, Kayser C, Logothetis NK, Panzeri S (2013). Modelling and analysis of local field potentials for studying the function of cortical circuits. Nat Rev Neurosci 14: 770-785.

Engel AK, Fries P, Singer W (2001). Dynamic predictions: oscillations and synchrony in top-down processing. Nat Rev Neurosci 2: 704-716.

Fernandez-Ruiz A, Herreras O (2013). Identifying the synaptic origin of ongoing neuronal oscillations through spatial discrimination of electric fields. Front Comput Neurosci 7: 5.

Fries P (2005). A mechanism for cognitive dynamics: neuronal communication through neuronal coherence. Trends Cogn Sci 9: $474-480$

George SA, Knox D, Curtis AL, Aldridge JW, Valentino RJ, Liberzon I (2013). Altered locus coeruleus-norepinephrine function following single prolonged stress. Eur J Neurosci 37: 901-909.

Gladstone GL, Parker GB, Malhi GS (2006). Do bullied children become anxious and depressed adults?: A cross-sectional investigation of the correlates of bullying and anxious depression. J Nerv Ment Dis 194: 201-208.
Gunnar MR, Wewerka S, Frenn K, Long JD, Griggs C (2009). Developmental changes in hypothalamus-pituitaryadrenal activity over the transition to adolescence: normative changes and associations with puberty. Dev Psychopathol 21: 69-85.

Halligan SL, Herbert J, Goodyer I, Murray L (2007). Disturbances in morning cortisol secretion in association with maternal postnatal depression predict subsequent depressive symptomatology in adolescents. Biol Psychiatry 62: 40-46.

Jacinto LR, Reis JS, Dias NS, Cerqueira JJ, Correia JH, Sousa N (2013). Stress affects theta activity in limbic networks and impairs novelty-induced exploration and familiarization. Front Behav Neurosci 7: 127

Jodo E, Chiang C, Aston-Jones G (1998). Potent excitatory influence of prefrontal cortex activity on noradrenergic locus coeruleus neurons. Neuroscience 83: 63-79.

Lestienne R, Herve-Minvielle A, Robinson D, Briois L, Sara SJ (1997). Slow oscillations as a probe of the dynamics of the locus coeruleus-frontal cortex interaction in anesthetized rats. J Physiol 91: 273-284.

Lesting J, Daldrup T, Narayanan V, Himpe C, Seidenbecher T, Pape HC (2013). Directional theta coherence in prefrontal cortical to amygdalo-hippocampal pathways signals fear extinction. PLoS One 8: e77707.

Lupien SJ, McEwen BS, Gunnar MR, Heim C (2009). Effects of stress throughout the lifespan on the brain, behaviour and cognition. Nat Rev Neurosci 10: 434-445.

McCormick CM, Mathews IZ (2007). HPA function in adolescence: role of sex hormones in its regulation and the enduring consequences of exposure to stressors. Pharmacol Biochem Behav 86: $220-233$.

McCormick CM, Mathews IZ (2010). Adolescent development, hypothalamic-pituitary-adrenal function, and programming of adult learning and memory. Prog Neuropsychopharmacol Biol Psychiatry 34: 756-765.

McCormick CM, Mathews IZ, Thomas C, Waters P (2010). Investigations of HPA function and the enduring consequences of stressors in adolescence in animal models. Brain Cogn 72: 73-85.

Miczek KA (1979). A new test for aggression in rats without aversive stimulation: differential effects of d-amphetamine and cocaine. Psychopharmacology (Berl) 60: 253-259.

Morrison JH, Molliver ME, Grzanna R (1979). Noradrenergic innervation of cerebral cortex: widespread effects of local cortical lesions. Science 205: 313-316.

Novick AM, Miiller LC, Forster GL, Watt MJ (2013). Adolescent social defeat decreases spatial working memory performance in adulthood. Behav Brain Funct 9: 39.

Rygula R, Abumaria N, Flugge G, Fuchs E, Ruther E, HavemannReinecke U (2005). Anhedonia and motivational deficits in rats: impact of chronic social stress. Behav Brain Res 162: $127-134$

Sara SJ, Herve-Minvielle A (1995). Inhibitory influence of frontal cortex on locus coeruleus neurons. Proc Natl Acad Sci USA 92: 6032-6036.

Sebastian C, Viding E, Williams KD, Blakemore SJ (2010). Social brain development and the affective consequences of ostracism in adolescence. Brain Cogn 72: 134-145.

Snyder K, Wang WW, Han R, McFadden K, Valentino RJ (2012). Corticotropin-releasing factor in the norepinephrine nucleus, locus coeruleus, facilitates behavioral flexibility. Neuropsychopharmacology 37: 520-530.

Snyder K, Barry M, Plona Z, Ho A, Zhang XY, Valentino RJ (2015). The impact of social stress during adolescence or adulthood and coping strategy on cognitive function of female rats. Behav Brain Res 286: 175-183.

Valentino RJ, Wehby RG (1988). Morphine effects on locus coeruleus neurons are dependent on the state of arousal and 
availability of external stimuli: Studies in anesthetized and unanesthetized rats. J Pharmacol Exp Ther 244: 1178-1186.

Valentino RJ, Van Bockstaele E (2008). Convergent regulation of locus coeruleus activity as an adaptive response to stress. Eur J Pharmacol 583: 194-203.

Wood SK, Walker HE, Valentino RJ, Bhatnagar S (2010). Individual differences in reactivity to social stress predict susceptibility and resilience to a depressive phenotype: role of corticotropinreleasing factor. Endocrinology 151: 1795-1805.
Wood SK, McFadden KV, Grigoriadis D, Bhatnagar S, Valentino RJ (2012). Depressive and cardiovascular disease comorbidity in a rat model of social stress: a putative role for corticotropinreleasing factor. Psychopharmacology (Berl) 222: 325-336.

Wood SK, Zhang XY, Reyes BA, Lee CS, Van Bockstaele EJ, Valentino RJ (2013). Cellular adaptations of dorsal raphe serotonin neurons associated with the development of active coping in response to social stress. Biol Psychiatry 73: 1087-1094.

Supplementary Information accompanies the paper on the Neuropsychopharmacology website (http://www.nature.com/npp) 